\title{
Diversity and zonation of mangrove flora in Belitung Island, Indonesia
}

\author{
AFRI IRAWAN ${ }^{1}$, TATIK CHIKMAWATI ${ }^{2, \bullet}$, SULISTIJORINI $^{2}$ \\ ${ }^{1}$ Plant Biology Graduate Program, Department of Biology, Faculty of Mathematics and Natural Sciences, Institut Pertanian Bogor. Jl. Agatis, Dramaga, \\ Bogor 16680, West Java, Indonesia \\ ${ }^{2}$ Department of Biology, Faculty of Mathematics and Natural Sciences, Institut Pertanian Bogor. Jl. Agatis, Dramaga, Bogor 16680, West Java, \\ Indonesia. Tel./fax.: +62-251-8622833, `email: tatikch@apps.ipb.ac.id
}

Manuscript received: 18 January 2021. Revision accepted: 30 April 2021.

\begin{abstract}
Irawan A, Chikmawati T, Sulistijorini. 2021. Diversity and zonation of mangrove flora in Belitung Island, Indonesia. Biodiversitas 22: 2981-2992. Mangrove is an important ecosystem located in the coastal tidal zone in tropical and sub-tropical regions. This ecosystem is characterized by the presence of plant species that can survive in brackish and inundated zone of seawater. There are many mangrove forests in Belitung Island, Indonesia, but the data of mangrove flora diversity and zonation in this island have not been described. This study aims to reveal the diversity and analyze the zonation of mangrove flora in Belitung Island. This research was conducted using a cruising method to explore the diversity of mangrove flora species and a line transect to analyze the zonation of mangrove flora. The exploration locations for diversity of mangrove flora were Kuale Tambak Beach, Asam Beach, Belitung Mangrove Park Area, Manggar River, Sentigi Beach, Sabong Beach and Kembiri Beach. Mangrove flora zonation was observed in Asam Beach (north), Kembiri Beach (south) and Kuale Tambak Beach (east). The result showed that mangrove flora in Belitung Island consisted of 12 families, 16 genera and 24 species. Species found were dominated by 8 species from the Rhizophoraceae family with the largest number of species were from the genera of Bruguiera and Rhizophora. The species of Bruguiera included Bruguiera gymnorhiza, Bruguiera sexangula, Bruguiera cylindrica and Bruguiera parviflora. Meanwhile, the Rhizophora species that have been found were Rhizophora apiculata, Rhizophora mucronata and Rhizophora stylosa. The Kembiri beach had the highest number of species of mangrove flora with 18 species. The zonation of mangrove flora at each research location showed a different zonation pattern. The mangrove vegetation on Kulae Tambak Beach was behind the Casuarina equisetifolia vegetation, while the mangrove vegetation on the Asam and Kembiri Beaches directly faced the sea. The difference in zonation patterns is likely caused by local topography on Belitung Island, substrate conditions and salinity factors on the mangroves of Belitung Island.
\end{abstract}

Keywords: Belitung, diversity, flora, mangrove, zonation

\section{INTRODUCTION}

Mangroves are important ecosystems in the world that grow in tropical and subtropical regions. The world's mangrove ecosystem was estimated at about 15.2 million hectares in 2005 (FAO 2007). Mangrove forests are only found in a few countries in the world in which Indonesia, Australia, Brazil, Nigeria and Mexico alone contribute to $48 \%$ of the world's mangrove area (FAO 2007). Indonesia has 2.5 million hectares of mangroves area or equal to $19 \%$ of the global mangroves, making Indonesia as the country with the largest mangrove ecosystems (FAO 2007; Giesen et al. 2007).

Mangrove flora has different characteristics from most terrestrial plants. The root system of mangrove plants is adapted to grow on mangrove ecosystem, which is periodically inundated by brackish water. Some types of roots are anchor roots, pencil roots, knee roots or buttress roots (Krauss et al. 2014; Hendy and Cragg 2017; Martinez and Buot Jr. 2018). In addition, the types of fruit in mangrove plants are vivipary, crypto-vivipary or nonvivipary (Karyamsetty 2017).

The dominant flora found in mangrove forests is the family of Rhizophoraceae. The genera of Rhizophoraceae include Rhizophora, Bruguiera, Ceriops and Kandelia. Apart from the Rhizophoraceae, there is also the family of
Acanthaceae, Apocynaceae, Arecaceae, Combretaceae, Euphorbiaceae, Lythraceae, Meliaceae, Myrtaceae, Primulaceae and Rubiaceae (Noor et al. 2012). In addition, there is also a naturally hybridized mangrove species, Bruguiera $\mathrm{x}$ dungarra which is a hybridization of Bruguiera exaristata and Bruguiera gymnorhiza, which is found in Northeast Australia (Duke and Kudo 2018).

The species of mangrove tends to form zonation pattern in which each zone from the seashore to the inland has different mangrove species (Tomlinson 1994). This zonation is formed due to the ability of the mangrove flora to grow on a specific type of substrate. These mangrove species grow specifically on the type of mud, sand, or rocky mud substrate, as well as their sensitivity to salinity and tides (Bunt and Williams 1981). Different water salinity and soil type in each zone will form the distribution pattern of mangrove plants (Islam et al. 2019). For example, Sonneratia prefers sandy and inundated substrates (Chen et al. 2013), while Avicennia is more tolerant to a wide salinity range (Basyuni et al. 2012; Martinez and Buot Jr 2018), and Rhizophora prefers muddy and inundated substrates (Hoppe-Speer et al. 2011).

Besides the differences in species across zones, the floristic composition of mangrove species also varies in different locations. For example, in the Mai Po, Hong Kong mangrove vegetation is dominated by Kandelia obovata, 
Avicennia marina and Acanthus ilicifolius (Liu et al. 2018). The mangrove forest in Cengkrong Beach, East Java is dominated by Sonneratia alba, Rhizophora mucronata, Bruguiera gymnorhiza and Ceriops tagal (Mughofar et al. 2018). Mangrove vegetation in Metinaro, Timor Leste is dominated by Rhizophora apiculata (Costa et al. 2019). The species of mangroves found in Tanjung Panjang Nature Reserve, Gorontalo are Ceriops tagal, Rhizophora mucronata and Rhizophora stylosa (Baderan et al. 2018). Mangrove ecosystem in Tanamon Beach, North Sulawesi is dominated by Sonneratia alba, Avicennia marina and Xylocarpus granatum (Yuningsih et al. 2013). The composition and stands of mangroves in Alas Purwo National Park are dominated by Bruguiera cylindrica, Rhizophora mucronata, Avicennia officinalis and Xylocarpus moluccensis (Heriyanto and Subiandono 2012). The mangrove area on the coast of Sebatik Island, East Kalimantan is dominated by Sonneratia alba, Rhizophora apiculata, Avicennia alba and Rhizophora mucronata (Ardiansyah et al. 2012).

Several studies of mangrove flora have been carried out in the Province of Bangka-Belitung Islands, Indonesia. Research on the potential and composition of mangroves flora in the Nasik Strait, Belitung District obtained eight species of mangrove flora (Firmansyah et al. 2013). The diversity and distribution of mangroves in the small islands of Manggar Sub-district, East Belitung District are reported to be nine species of mangrove flora (Ridho et al. 2015). A study of mangrove distribution in Pongok Island, South Bangka shows that mangrove vegetation in the area is dominated by Rhizophora, Avicennia, Sonneratia and Bruguiera (Umroh et al. 2016). The mangrove vegetation in Sebongkok Island, East Belitung consists of seven species and dominated by Rhizophora spp. (Akhrianti 2016). In the northern adjacent islands of Tambelan and Serasan, Bruguiera gymnorhiza and Rhizophora stylosa are the dominant species (Ulumuddin and Setyawan 2017).
While several studies on mangroves have been conducted in Bangka-Belitung Islands, the mangrove flora in Belitung Island has not been studied taxonomically and ecologically. This study aims to describe the diversity and analyze the zonation of mangrove flora species in Belitung Island by taxonomical and ecological studies. Several locations of mangrove forests in Belitung Island will be proposed as conservation areas. The data of the species of mangrove flora in this study can be used to improve the conservation status of the location. The mangrove data can also be used as a database of mangrove flora diversity for development of flora conservation in Belitung Island, the Province of Bangka-Belitung Islands.

\section{MATERIALS AND METHODS}

\section{Study period and area}

Field exploration was carried out from October 2019 to November 2019 in several mangrove forest locations in Belitung Island, the Province of Bangka-Belitung Island, Indonesia (Figure 1). The identification of mangrove flora species and data analysis was carried out from November 2019 to March 2020 at the Plant Resources and Ecology Laboratory of the Department of Biology, Mathematics and Natural Science Faculty, IPB University, Bogor, Indonesia.

The exploration locations for mangrove flora in Belitung Island were Kuale Tambak Beach (Sukamandi, Damar Sub-district), Asam Beach (Aik Kelik, Damar Subdistrict), Belitung Mangrove Park Area (Juru Seberang, Tanjung Pandan Sub-district), Manggar River (Mekar Jaya, Manggar Sub-district), Sentigi Beach (Dukong, Simpang Pesak Sub-district), Sabong Beach (Tanjung Rusa, Membalong Sub-district) and Kembiri Beach (Kembiri, Membalong Sub-district). The detailed information of each sampling location is presented in Table 1.

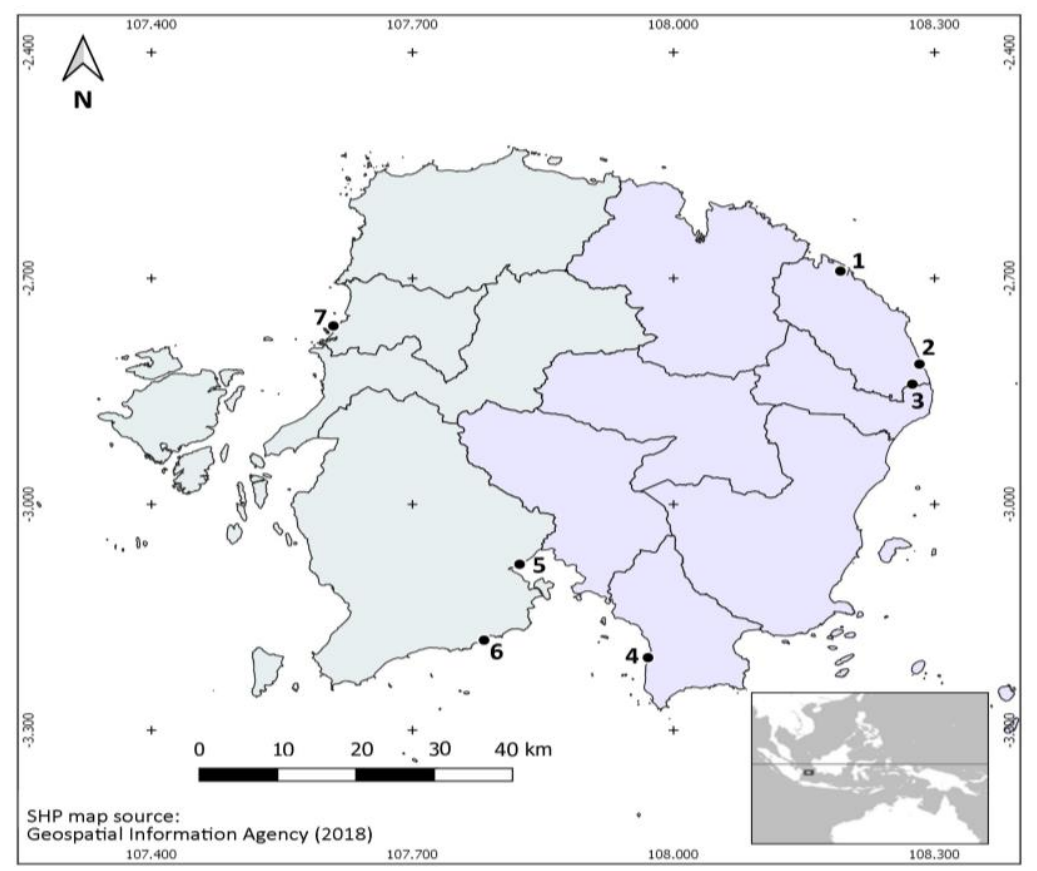

Figure 1. Map of sampling location points in Belitung Island, Indonesia (showed by dot and number 1-7) 
Table 1. The coordinate location of research sampling in Belitung Island, Indonesia

\begin{tabular}{|c|c|c|c|c|}
\hline \multirow{2}{*}{ Sampling location } & \multirow{2}{*}{ Sub-district } & \multirow{2}{*}{ Conditions } & \multicolumn{2}{|c|}{ Coordinate position } \\
\hline & & & Latitude (S) & Longitude (E) \\
\hline Asam beach & Damar & Primary forest, natural tourism and fishing activity & $2^{\circ} 41 ' 25.40^{\prime \prime}$ & $108^{\circ} 1130.43^{\prime \prime}$ \\
\hline Kuale Tambak beach & Damar & Natural tourism and fishing activity & $2^{\circ} 48 ' 49.86 "$ & $108^{\circ} 16^{\prime} 57.44^{\prime \prime}$ \\
\hline Manggar river & Manggar & River near mines and settlements & $2^{\circ} 50^{\prime} 25.71^{\prime \prime}$ & $108^{\circ} 16 \prime 28.02^{\prime \prime}$ \\
\hline Sentigi beach & Simpang Pesak & Natural tourism & $3^{\circ} 12^{\prime} 12.76^{\prime \prime}$ & $107^{\circ} 58^{\prime} 14.54^{\prime \prime}$ \\
\hline Sabong beach & Membalong & Primary forest & $3^{\circ} 10^{\prime} 49.32^{\prime \prime}$ & $107^{\circ} 466^{\prime} 56.10^{\prime \prime}$ \\
\hline Kembiri beach & Membalong & Primary forest & $3^{\circ} 4^{\prime} 46.45^{\prime \prime}$ & $107^{\circ} 49^{\prime} 23.27^{\prime \prime}$ \\
\hline Belitung Mangrove Park & Tanjung Pandan & Restoration forest and natural tourism & $2^{\circ} 45^{\prime} 47.14^{\prime \prime}$ & $107^{\circ} 366^{\prime} 32.78^{\prime \prime}$ \\
\hline
\end{tabular}

\section{Data collection procedure}

Assessment of mangrove diversity

We conducted the cruising method to collect field data and herbarium samples to determine the diversity of mangrove flora (Rugayah et al. 2004). Exploration paths in each mangrove forest include the edge of the mangrove forest adjacent to the open sea, the middle part of the mangrove forest and part of the border between the mangrove forest and the mainland forest. The cruising line schematic is shown in Figure 2.A. Each individual mangrove plant was taken several twigs consisting of leaves, flowers and fruit to make herbarium specimens. Some characteristics recorded in the field include: root type, flower color and the other part that allow discoloration when it was made into a herbarium.

\section{Assessment of mangrove zones}

We used the line transect method to study the zonation of mangrove plants (Phillips 1959). Mangrove species zonation was observed in Asam Beach (north), Kembiri Beach (south) and Kuale Tambak Beach (east). Transect establishment was carried out perpendicularly to the shore strandline (Figure 2.B). The length of the transect corresponds to the area of the mangrove forest. Transects on Asam Beach about 200 meters, Kembiri Beach about 150-300 meters, and Kuale Tambak Beach about 50-65 meters. The distance between the transects is about 150500 meters. Mangrove flora zonation was observed along these transects. Environmental variable data were measured at each research station (Figure 2.B). The measurement site consists of 3 stations of each beach, namely: station 1 , the station closest to the sea (front mangrove); station 2, the station in the middle of the mangrove; and station 3 , the farthest station from the sea (back mangrove). Soil samples were taken from each different substrate condition to analyze the substrate type. The measured abiotic data are salinity, water $\mathrm{pH}$, water temperature, air humidity, air temperature, wind velocity and light intensity.

\section{Species identification}

Identification of mangrove flora used Giesen et al. (2007) and Noor et al. (2012). Whereas, the identity of mangrove specimens collected from field were then confirmed with Herbarium Bogoriense (BO) specimens.

\section{Data analysis}

Diversity index values were calculated using the Shannon-Wiener diversity index (Magurran 1988). The percentage abundance of species was calculated by comparing the number of individuals of one species to the total number of individuals of all species.

The zonation of mangrove flora was described based on the species finding on each research transect. Analysis of the interaction of mangrove species with abiotic factors was done using Canonical Correspondence Analysis (CCA) with Monte Carlo permutation test in the software program Canoco for Windows 4.5.
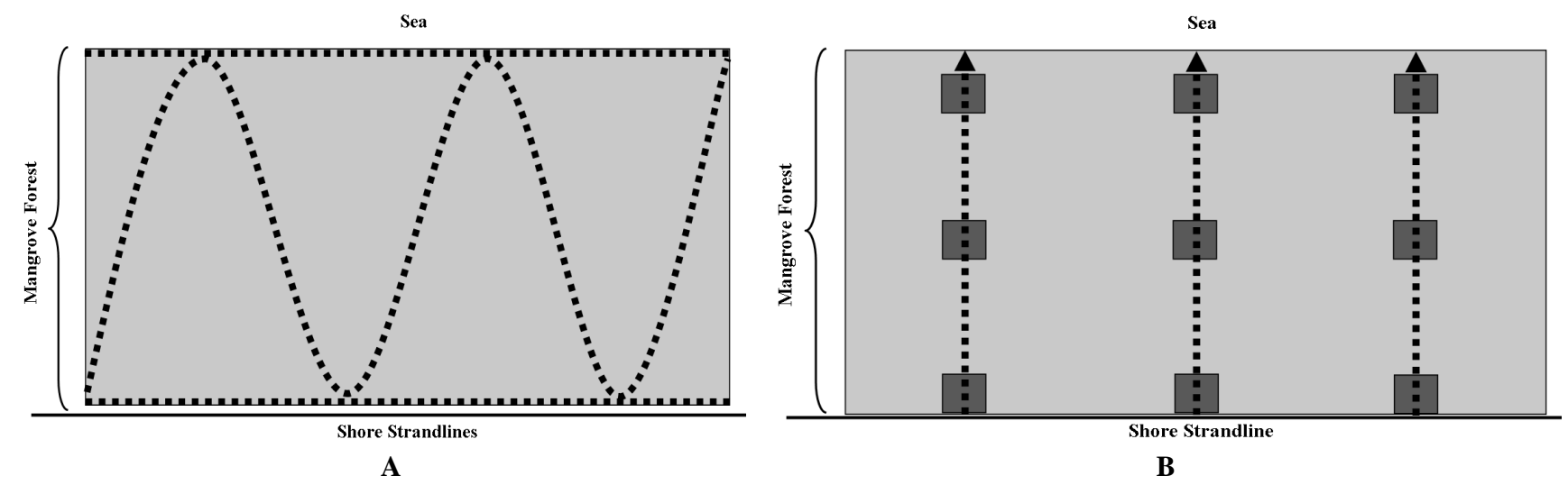

Figure 2. The methods of data collection applied in this study in Belitung Island, Indonesia: A. The cruising line scheme is shown as dotted line for diversity data collection; B. Line transect (dashed line) for zonation data collection and environmental data plot (square symbol) 


\section{RESULTS AND DISCUSSION}

\section{Diversity of mangrove flora}

The mangrove flora found in Belitung Island consisted of 12 families, 16 genera and 24 species. These species of mangrove flora were obtained from several locations in Belitung Island (Table 2). The largest family dominating the mangrove flora was Rhizophoraceae consisted of eight species. The genera with the most species are Bruguiera and Rhizophora.

The largest number of species of mangrove flora were found in Kembiri Beach consisted of 18 species. The diversity index value at this research station was 2.7 while that in the eastern region of Belitung Island (Kuale Tambak Beach) was 2.61 and on Asam Beach was 2.53. The values of the Shannon Wiener diversity index at all research stations were greater than 1 but less than $3\left(1<\mathrm{H}^{\prime}<3\right)$. The index value indicates that all research stations had a moderate category of diversity (Wilhm and Dorris 1968).

The species of mangrove flora with the highest to the lowest percentage of species abundance are presented in Figure 3. The mangrove flora species with the highest abundance percentage were Rhizophora apiculata, Rhizophora mucronata and Bruguiera gymnorhiza (Figure 4). The three species with the highest percentage of abundance are the members of the Rhizophoraceae, the largest plant family in the mangrove ecosystem. The abundance percentage of Rhizophora apiculata was $14.44 \%$, Rhizophora mucronata was $11.24 \%$ and Bruguiera gymnorhiza was $8.59 \%$. Rhizophora apiculata was found with the highest number of individuals at each research station. The species of mangrove flora with the lowest percentage of species abundance was Finlaysonia obovata. This climbing mangrove species was only found in Kembiri Beach as many as 2 individuals. Its abundance value was $0.18 \%$. Other species of mangrove flora with low abundance that were only found at one research station were Aegiceras corniculatum $(0.46 \%)$ and Bruguiera parviflora $(0.73 \%)$ in Kembiri Beach and Avicennia marina $(0.91 \%)$ in Belitung Mangrove Park.

The associate mangrove species were found in several mangrove ecosystems in rare stands in some areas including Sabong Beach, Kembiri Beach and Belitung Mangrove Park. There were four associate mangrove species found in Sabong Beach, namely Scaevola taccada, Planchonella obovata, Hibiscus tiliaceus and Cycas rumphii. Five associate mangrove species found in Kembiri Beach were Cerbera manghas, Intsia bijuga, Planchonella obovata, Cerbera odollam and Hibiscus tiliaceus. Several species of associate mangroves in the Belitung Mangrove Park consisted of Scaevola taccada, Hibiscus tiliaceus, Terminalia catappa and Pandanus tectorius.

Table 2. List of mangrove flora species in Belitung Island, Indonesia

\begin{tabular}{|c|c|c|c|c|c|c|c|c|}
\hline \multirow{2}{*}{ Family } & \multirow{2}{*}{ Species } & \multicolumn{7}{|c|}{ Site } \\
\hline & & PKT & PA & BMP & $\mathbf{S M}$ & PSt & $\mathbf{P S b}$ & PK \\
\hline Acanthaceae & Acanthus ilicifolius & & & & + & & + & + \\
\hline Acanthaceae & Avicennia alba & + & + & + & + & & & \\
\hline Acanthaceae & Avicennia marina & & & + & & & & \\
\hline Apocynaceae & Finlaysonia obovata & & & & & & & + \\
\hline Arecaceae & Nypa fruticans & + & + & + & + & & + & + \\
\hline Combretaceae & Lumnitzera littorea & + & + & + & & & + & + \\
\hline Combretaceae & Lumnitzera racemosa & + & & & + & + & + & \\
\hline Euphorbiaceae & Excoecaria agallocha & + & + & + & + & + & + & + \\
\hline Lythraceae & Pemphis acidula & + & + & + & + & + & & \\
\hline Lythraceae & Sonneratia alba & + & + & + & + & & & + \\
\hline Malvaceae & Heritiera littoralis & & + & & + & & + & + \\
\hline Meliaceae & Xylocarpus granatum & + & + & & & & + & + \\
\hline Primulaceae & Aegiceras corniculatum & & & & & & & + \\
\hline Pteridaceae & Acrostichum aureum & + & & + & + & + & & + \\
\hline Pteridaceae & Acrostichum speciosum & + & + & & + & + & + & + \\
\hline Rhizophoraceae & Bruguiera cylindrica & + & + & + & + & & & \\
\hline Rhizophoraceae & Bruguiera gymnorhiza & + & + & + & + & + & + & + \\
\hline Rhizophoraceae & Bruguiera parviflora & & & & & & & + \\
\hline Rhizophoraceae & Bruguiera sexangula & & & & & + & & + \\
\hline Rhizophoraceae & Ceriops tagal & + & + & & & & & + \\
\hline Rhizophoraceae & Rhizophora apiculata & + & + & + & + & + & + & + \\
\hline Rhizophoraceae & Rhizophora mucronata & + & + & + & + & + & + & + \\
\hline Rhizophoraceae & Rhizophora stylosa & + & + & + & & & & \\
\hline Rubiaceae & Scyphiphora hydrophyllacea & + & + & + & + & + & + & + \\
\hline Total (species) & & 17 & 16 & 14 & 15 & 10 & 11 & 18 \\
\hline Shannon-Wiener & & 2.61 & 2.53 & 2.49 & 2.51 & 2.19 & 2.4 & 2.7 \\
\hline
\end{tabular}

Notes: PKT. Kuale Tambak Beach; PA. Asam Beach; BMP. Belitung Mangrove Park; SM. Manggar River; PSt. Sentigi Beach; PSb. Sabong Beach; PK. Kembiri Beach; (+): present 


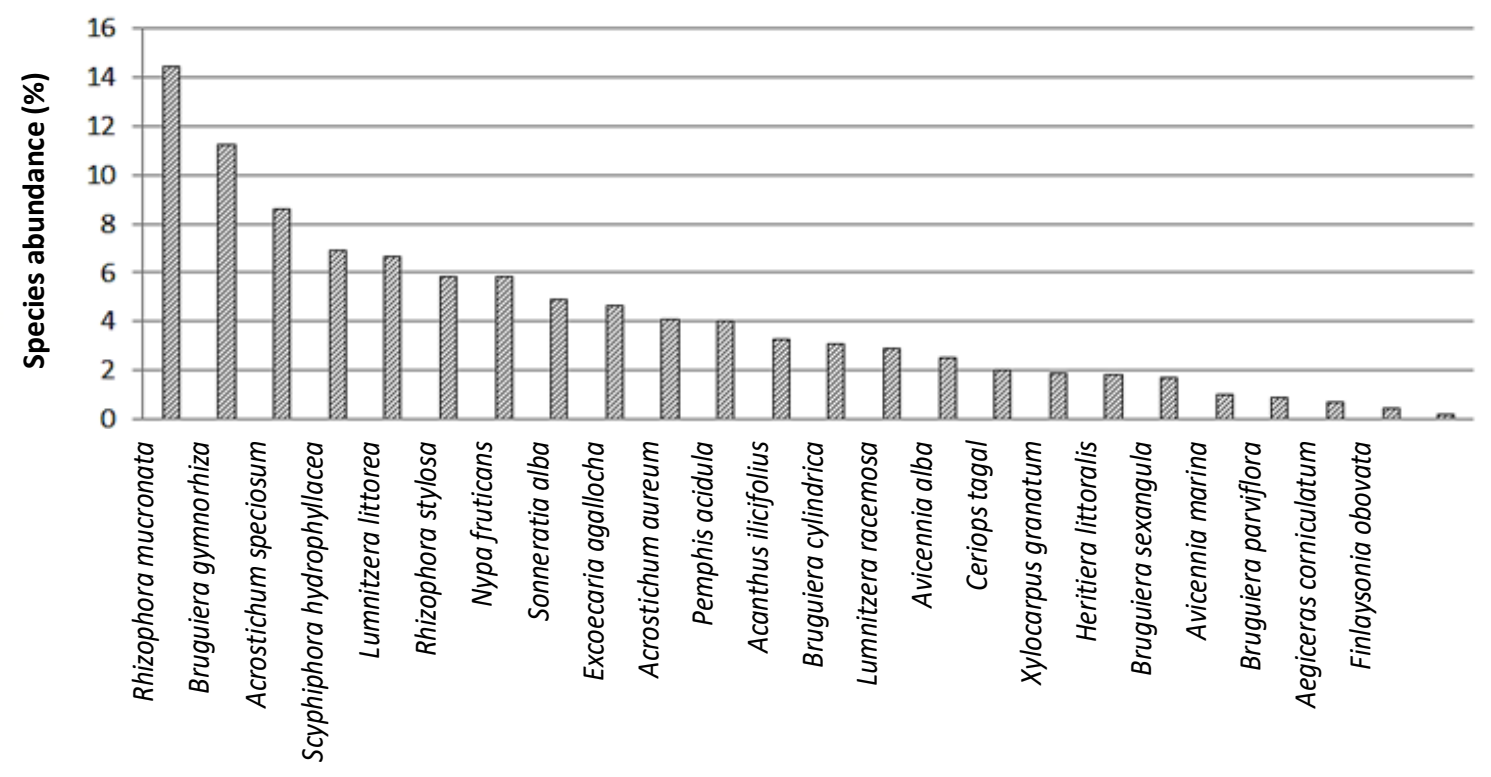

Figure 3. Species abundance percentage of mangrove flora
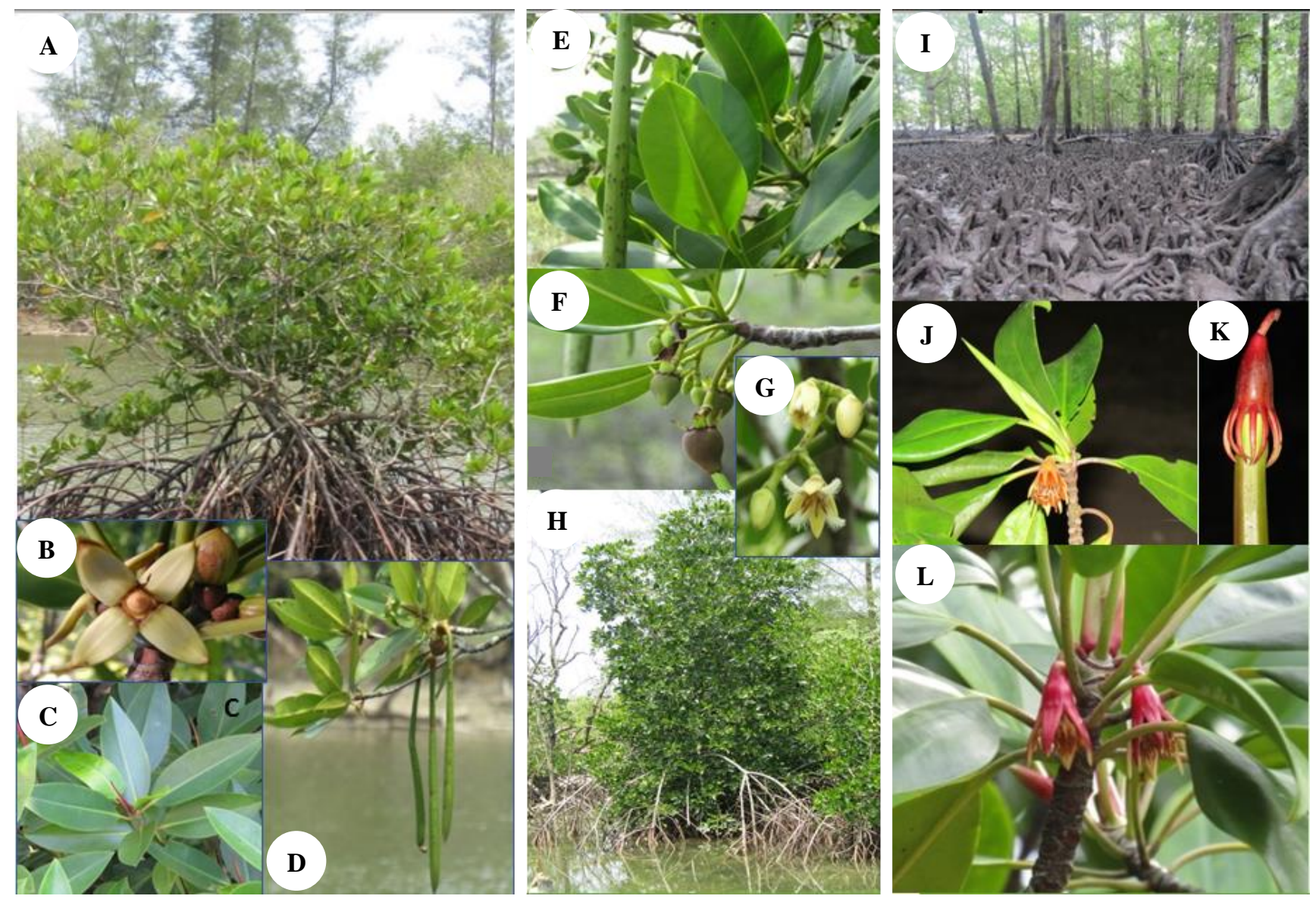

Figure 4. The mangrove species with the highest abundances found in all research sites in Belitung Island, Indonesia. A-D. Rhizophora apiculata, E-H. Rhizophora mucronata, I-L. Bruguiera gymnorhiza 
We provide an identification key and diagnostic description of mangrove flora in Belitung Island as described below.

\section{The identification key for mangrove flora in Belitung Island}

1. a. Leaves simple ...................................................... 2

b. Leaves compound ........................... 21

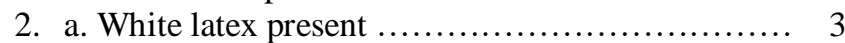

b. White latex absent ................................................... 4

3. a. Trees; leaves alternate, apex acuminate; flower unisexual ................................... Excoecaria agallocha b. Lianas; leaves opposite, apex rounded; flower bisexual ........................................ Finlaysonia obovata

4. a. Stipules present .................................................. 5 b. Stipules absent .................................................... 14

5. a. Stipules persistent ....... Scyphiphora hydrophyllacea b. Stipules caducous .................................................... 6

6. a. Inflorescences paniculate; flowers unisexual Heritiera littoralis b. Inflorescences solitary or cyme; flowers bisexual .... 7

7. a. Root systems knee root ........................................ 8 b. Root systems stilt root ........................................... 12

8. a. Leaf apex obtuse; sepals 5; petals 5 .... Ceriops tagal b. Leaf apex acuminate; sepals 6-14; petals 6-14 ........ 9

9. a. Flower compound; sepals 6-8; petals 6-8 .............. 10 b. Flower solitary; sepals $10-14$, petals $10-14$........... 11

10. a. Calyx lobes 1-2 cm long, reflexed

b. Calyx lobes 0,2-0,5 cm long, erect Bruguiera cylindrica

11. a. Calyx red; petal bristles present Bruguiera parviflora Bruguiera gymnorhiza

b. Calyx yellowish; petal bristles absent Bruguiera sexangula

12. a. Inflorescences 1-2 flowered; petals glabrous Rhizophora apiculata

b. Inflorescences 3 or more flowers; petals hairy ....... 13

13. a. Bark brownish, bark fragments square; leaf blade 7-9 cm long; inflorescences 3-4 flowered

Rhizophora mucronata b. Bark blackish, bark fragments irregularly; leaf blade 4-6 cm long; inflorescences 6-8 flowered

Rhizophora stylosa

14. a. Pneumatophore pencil roots present .. 15

b. Pneumatophore pencil roots absent ...................... 17

15. a. Leaf apex acuminate; sepals 4; petals 4, yellow .... 16 b. Leaf apex rounded; sepals 6-8; petals 6-8, white ........ Sonneratia alba

16. a. Inflorescences spike, flowers attached along the inflorescence stalk .................... Avicennia alba b. Inflorescences cyme, flowers clustered at the ends of the inflorescences Avicennia marina

17. a. Shrub; leaf margin spinose ......... Acanthus ilicifolius b. Tree; leaf margin entire ... 18

18. a. Flower solitary; sepals 6; petals 6 ... Pemphis acidula b. Flower compound; sepals 5; petals 5 . 19

19. a. Leaves alternate; inflorescences umbellate Aegiceras corniculatum b. Leaves spiral; inflorescences racemose or spike .... 20
20. a. Leaves oblanceolate; inflorescences racemose, terminal; petals red; stamens red; pistil red ... Lumnitzera littorea b. Leaves obovate; inflorescences spike, axillary; petals white; stamens white; pistil white

Lumnitzera racemosa

21. a. Young leaves curled up; reproduction with spore .. 22

b. Young leaves not curled up; reproduction with flower

................................................................... 23

22. a. Leaflet apex cuspidate ........... Acrostichuum aureum

b. Leaflet apex acute ............... Acrostichum speciosum

23. a. Leaves rosette; leaflets 50-100; apex acute

b. Leaves spiral; leaflet 2-6; apex obtuse Nypa fruticans Xylocarpus granatum

\section{Species diagnostic description}

Acanthus ilicifolius L., Sp. Pl. 639 (1753)

Shrub, up to $2 \mathrm{~m}$ tall. Pencil root pneumatophore absent. Stem erect, white latex absent. Stipules absent. Leaves simple, opposite, lanceolate, apex acuminate, margin spinose. Inflorescences racemose, terminal. Flower bisexual; sepals 4, green; petals 4, sympetalous, base white, mid to apex bluish. Fruits simple, capsular

\section{Acrostichum aureum L., Sp. Pl. 2:1069 (1753)}

Fern, up to $3 \mathrm{~m}$ tall. Leaves compound, pinnate; young leaves curled up; leaflet alternate, lanceolate; apex sterile leaflets cuspidate; apex fertile leaflet cuspidate, sporangium covers the abaxial surface when it matures.

\section{Acrostichum speciosum Willd., Sp. Pl. 5(1-2):117 (1810)}

Fern, up to $2 \mathrm{~m}$ tall. Stem forming rhizomes. Leaves compound, pinnate; young leaves curled up; leaflet alternate, lanceolate; apex sterile leaflet acute; apex fertile leaflet acute, sporangium covers the abaxial surface when it matures.

Aegiceras corniculatum (L.) Blanco, Fl. Filip. 79 (1837)

Small tree, up to $8 \mathrm{~m}$ tall. Pencil root pneumatophores absent. Stem erect, white latex absent; bark brownish; young stem reddish. Stipules absent. Leaves simple, alternate, obovate, apex rounded, margin entire, adaxial green glossy, abaxial pale. Inflorescences compound, umbellate, terminal. Flower bisexual; calyx green, contorted; sepals 5; corolla white; petal 5; stamens 5, white; pistil solitary, white. Fruits simple, cryptoviviparous, hypocotyl grows towards the fruit stalk.

\section{Avicennia alba Blume, Bijdr. 821 (1826)}

Tree, up to $25 \mathrm{~m}$ tall. Pencil root pneumatophores present. Stem erect, white latex absent; bark grayish, not flaky. Stipules absent. Leaves simple, opposite, lanceolate, apex acuminate, adaxial green glossy, abaxial whitish. Inflorescences spike; flower units attached along the stalk. Flower bisexual; calyx green; sepals 4; corolla yelloworange; petals 4; stamens 4, yellow; pistil solitary. Fruits simple, green-yellowish, viviparous, cone shapes. 
Avicennia marina (Forssk.) Vierh., Wien. Math.-Naturwiss. K1. 71:435 (1907)

Tree, up to $8 \mathrm{~m}$ tall. Pencil root pneumatophores present. Stem erect, white latex absent; bark grayish, not flaky. Stipules absent. Leaves simple, opposite, elliptical, apex acuminate, adaxial green glossy, abaxial whitish. Inflorescences cyme; flowers clustered at the end of the stalk. Flower bisexual; calyx green; sepals 4; corolla yelloworange; petals 4; stamens 4, yellow; pistil solitary. Fruits simple, green-yellowish, viviparous, cone to round shape.

Bruguiera cylindrica (L.) Blume, Enum. Pl. Javae 1:91 (1827)

Tree, up to $10 \mathrm{~m}$ tall. Knee root pneumatophores present. Stem erect, white latex absent; bark smooth and grayish. Stipules present, caducous. Leaves simple, decussate, elliptical, apex acuminate. Inflorescences cyme, axillary 2-3 flowered. Flower bisexual; calyx green, sulcate, calyx lobes 1-2 cm long, reflexed; sepals 6-8, rigid persistent; corolla white; petals 6-8, hairy, caducous, apex bristly; stamens free; pistil solitary. Fruits simple, viviparous; hypocotyl cylindrical, dangling.

\section{Bruguiera gymnorhiza (L.) Lamk., Encycl. Meth. Bot.} 4:696 (1798)

Tree, up to25 $\mathrm{m}$ tall. Knee root pneumatophores present. Stem erect, white latex absent; bark brownblackish, lenticellate. Stipules present, caducous. Leaves simple, decussate, elliptical, apex acuminate. Flower solitary, axillary bisexual; calyx red; sepals 10-14, rigid, persistent; corolla yellowish; petals 10-14, hairy, caducous, apex bristly; stamen free; pistil solitary. Fruits simple, viviparous; hypocotyl cylindrical, dangling.

\section{Bruguiera parviflora (Roxb.) Wight \& Arn. ex Griff.,} Trans. Med. Soc. Calcutta 8:10 (1836)

Tree, up to $25 \mathrm{~m}$ tall. Knee root pneumatophores present. Stem erect, white latex absent; bark smooth, grayish. Stipules present, caducous. Leaves simple, decussate, elliptical, apex acuminate. Inflorescences cyme, axillary, 3-6 flowered. Flowers bisexual; calyx yellowgreenish, calyx lobes $0,2-0,5 \mathrm{~cm}$ long, erect; sepals 6-8, persistent; corolla yellow; petals 6-8, caducous, apex bristly; stamens free; pistil solitary. Fruits simple, viviparous; hypocotyl cylindrical, smooth, dangling.

Bruguiera sexangula (Lour.) Poir., Encycl. Suppl. 4:262 (1816)

Tree, up to $20 \mathrm{~m}$ tall. Knee root pneumatophores present. Stem erect, white latex absent; bark graybrownish, lenticellate. Stipules present, caducous. Leaves simple, decussate, elliptical, apex acuminate. Flower solitary, axillary, bisexual; calyx yellow to orange; sepals 10-14, rigid, persistent; corolla yellowish; petal 10-14, hairy, caducous; apex not bristly; stamens free; pistil solitary. Fruits simple, viviparous; hypocotyl cylindrical.

Ceriops tagal (Perr.) C.B.Rob., Philipp. J. Sci. C 3:306 (1908)

Tree, up to $10 \mathrm{~m}$ tall. Knee root pneumatophores present. Stem erect, white latex absent; bark light grayish. Stipules present, caducous. Leaves simple, decussate, oblanceolate, apex obtuse. Inflorescences cyme, axillary, 4-
10 flowered. Flowers bisexual; calyx green-yellowish; sepals 5, rigid, persistent; corolla white-brownish; petals 5, caducous; stamens free; pistil solitary. Fruits simple, viviparous; calyx lobes on fruits reflexed; hypocotyl clavate, ridged, sulcate.

\section{Excoecaria agallocha L., Syst. Nat. ed.10. 2:1288 (1759)}

Tree, up to $15 \mathrm{~m}$ tall. Stem erect, white latex present; bark smooth, grayish. Leaves simple, alternate, elliptical, apex acuminate. Inflorescences spike, dioecious, unisexual. Male flowers apetalous; sepals 3, very small, about 0,5-1 $\mathrm{mm}$; stamens 3. Female flowers apetalous; sepals 3. very small, about 0,5-1 mm; carpels 3, ovary trilocular. Fruits simple, 3 locules.

\section{Finlaysonia obovata Wall. Pl. Asiat. Rar. 2: 48 (1831)}

Lianas. Stem climbing, white latex present. Leaves simple, opposite, obovate, apex rounded. Inflorescences cyme, axillary. Flowers bisexual; sepals 5; petals 5, upper hairy; stamens 5, epipetalous; carpels 2, apocarpous. Fruits aggregate, follicle.

\section{Heritiera littoralis Aiton, Hort. Kew. 3:546 (1789)}

Tree, up to $20 \mathrm{~m}$ tall. Buttress root present. Stem erect; bark grayish, white latex absent. Stipules present, caducous. Leaves simple, alternate, elliptical, apex acute. Inflorescences paniculate, unisexual. Male flowers pinkish to purplish; perianth 5 lobes; stamens monadelphous; anthers 5. Female flowers pinkish to purplish; perianth 5 lobes; pistil solitary, stigma 5 lobes. Fruits simple, greenbrownish; seed solitary.

Lumnitzera littorea (Jack) Voigt, Hort. Suburb. Calcutt. 39 (1845)

Tree, up to $35 \mathrm{~m}$ tall. Knee root pneumatophores absent. Stem erect, white latex absent; bark blackish; young stem red. Stipules absent. Leaves simple, spiral, oblanceolate, succulent, apex rounded, margin entire. Inflorescences racemose, terminal. Flowers bisexual, produce nectar; calyx green; sepals 5; corolla red; petals 5; stamens 10, red; pistil solitary, red. Fruits simple, ellipsoidal.

Lumnitzera racemosa Willd., Neue. Schriften. Ges. Naturf. Freunde. Berlin 4:187 (1803)

Small tree, up to $8 \mathrm{~m}$ tall. Knee root pneumatophores absent. Stem erect, white latex absent; bark brownish; young stem grayish. Stipules absent. Leaves simple, spiral, obovate, succulent, apex obtuse, margin entire. Inflorescences spike, axillary. Flowers bisexual, produce nectar; calyx green; sepals 5; corolla white; petals 5; stamens 10, white; pistil solitary, white. Fruits simple, ellipsoidal.

\section{Nypa fruticans Wurmb, Verh. Batav. Genootsch. Kunsten 1:349 (1779)}

Palms, up to $8 \mathrm{~m}$ tall. Stem short close to the substrate. Leaves compound, pinnate, rosette; leaflets 50-100, alternate, linear, apex acute. Inflorescences compound, unisexual. Fruits compound, brown, fibrous, shelled. 
Pemphis acidula J.R. Forst. \& G. Forst., Char. Gen. Pl. 34 (1775)

Small tree, up to $8 \mathrm{~m}$ tall. White latex absent; bark brownish, flaking. Stipules absent. Leaves simple, decussate, lanceolate, apex acuminate, margin entire. Flower solitary, axillary, bisexual; calyx green; sepals 6; corolla white; petals 6 , thin; stamens 12 ; pistil solitary. Fruits simple, capsular.

Rhizophora apiculata Blume, Enum. Pl. Javae 1:91 (1827)

Tree, up to $20 \mathrm{~m}$ tall. Stilt roots present. Stem erect, white latex absent; bark white-brownish. Stipules present, caducous. Leaves simple, decussate, elliptical, apex mucronate. Inflorescences solitary to 2 flowered, axillary. Flowers bisexual; calyx green-brownish; sepals 4, rigid, persistent; corolla white; petals 4 , thin, glabrous, caducous; stamens 12, caducous; pistil solitary. Fruits simple, viviparous; hypocotyl clavate, elongate, dangling.

Rhizophora mucronata Lamk., Encycl. 6:189 (1804)

Tree, up to $25 \mathrm{~m}$ tall. Stilt roots present. Stem erect, white latex absent; bark brownish; bark segments squared. Stipules present, caducous. Leaves simple, decussate, elliptical, 7-9 cm long, apex mucronate. Inflorescences cyme, axillary, 3-4 flowered. Flowers bisexual; calyx green; sepals 4, rigid, persistent; corolla white; petals 4, thin, hairy, caducous; stamens 8, caducous; pistil solitary. Fruits simple, viviparous; hypocotyl clavate, elongate, dangling.

\section{Rhizophora stylosa Griff., Not. Pl. Asiat. 4:665 (1854)}

Tree, up to $15 \mathrm{~m}$ tall. Stilt roots present. Stem erect, white latex absent; bark blackish; bark segments irregularly. Stipules present, caducous. Leaves simple, decussate, elliptical, 4-6 cm long, apex mucronate. Inflorescences cyme, axillary, 6-8 flowered. Flowers bisexual; calyx yellow-greenish; sepals 4, rigid, persistent; corolla white; petals 4, thin, hairy, caducous; stamens 8, caducous; pistil solitary. Fruits simple, viviparous; hypocotyl clavate, elongate, dangling.

\section{Scyphiphora hydrophyllacea C.F.Gaertn., Suppl. Carp. 91} (1806)

Small tree, up to $5 \mathrm{~m}$ tall. Stem erect, white latex absent; bark brownish; young stem reddish. Stipules present, persistent on interpetiolar. Leaves simple, opposite, obovate, apex rounded. Inflorescences cyme, 5-10 flowered. Flowers bisexual; sepals 4, green, synsepalous; petals 4, white, sympetalous; stamens 4, epipetalous; pistil solitary; stigma ramified. Fruits simple, cylindrical, sulcate.
Sonneratia alba Sm., Cycl. 33(I): Sonneratia no. 2 (1816)

Tree, up to $35 \mathrm{~m}$ tall. Pencil root pneumatophore present. Stem erect, white latex absent; bark white-grayish, flaking. Leaves simple, opposite, obovate, apex rounded. Inflorescences axillary, 1-3 flowered. Flowers bisexual; calyx green, inner reddish; sepals 6-8; corolla white, caducous; petals 6-8; stamens white, numerous, free; pistil solitary. Fruits simple, apple-like.

Xylocarpus granatum J.Koenig, Naturforscher (Halle) 20:2 (1784)

Tree, up to $30 \mathrm{~m}$ tall. Buttress root present. Stem erect; bark yellow-brownish, flaking. Leaves compound, pinnate, spiral; leaflets 2-6, oblong, apex obtuse. Inflorescences cyme, axillary, 8-15 flowered. Flowers bisexual; calyx yellow-greenish; sepals 4; corolla white, tubular; petals 4, sympetalous; stamens white, epipetalous; pistil solitary. Fruits $15-25 \mathrm{~cm}$ diameter; carpel brownish, 0,5-1,5 cm thick; seeds 8-16, irregular pyramid-like.

\section{Environmental conditions of mangrove ecosystem in \\ Belitung Island}

Substrate is a media for plant to grow. In mangrove ecosystem, there are several substrate conditions that differ from substrate of other ecosystem types. The substrate in the mangrove ecosystem is formed through the accumulation of sediment materials which are retained by the roots of mangrove plants. The type of substrate is a mixture of mud, sand and coral or clay with different ratios in each zone (Table 3).

The mangrove ecosystem is a kind of muddy substrate resulted from sedimentation. The sedimentation mud mixes with other materials, such as sand, clay or coral, then forms a variety of mangrove substrates (Table 3). The substrate of mangrove in Kuale Tambak Beach was sandy mixed with some mud. In Asam Beach, the mangrove substrate consisted of sand, coral and some mud. The substrate of the mangrove ecosystem of all stations in Kembiri Beach was dominated by mud with the depth of the mud at station 1 reached $100 \mathrm{~cm}$. There was a low portion of sand at station 1, but at stations 2 and 3, the mud has formed a dense and solid substrate by mixing with clay soil. The high proportion of mud in the Kembiri Beach is influenced by the flow of the Kembiri River which ends in Kembiri Beach. The width of the estuary of the Kembiri River reaches 365 meters. This substrate condition is different from the mangrove ecosystem in Sebongkok Island, East Belitung which is only dominated by sand (Akhrianti 2016).

Table 3. Type of mangrove substrate at each research station in Belitung Island, Indonesia

\begin{tabular}{|c|c|c|c|c|c|c|c|c|c|}
\hline \multirow{2}{*}{ Substrate type } & \multicolumn{3}{|c|}{ Kuale Tambak } & \multicolumn{3}{|c|}{ Asam } & \multicolumn{3}{|c|}{ Kembiri } \\
\hline & st1 & st2 & st3 & st1 & st2 & st3 & st1 & st2 & st3 \\
\hline Muddy & - & $*$ & $* *$ & & $*$ & $*$ & $* * * * *$ & $* * * * *$ & $* * * *$ \\
\hline Sandy & $* * * * * *$ & $* * * * *$ & $* * * *$ & $* * * *$ & $* * * *$ & $* * * * *$ & $*$ & - & - \\
\hline Coral & - & - & - & $* *$ & $*$ & - & - & - & - \\
\hline Clay & - & - & - & - & - & - & - & $*$ & $* *$ \\
\hline
\end{tabular}

Notes: st. station; (*). Present; (-). absent 


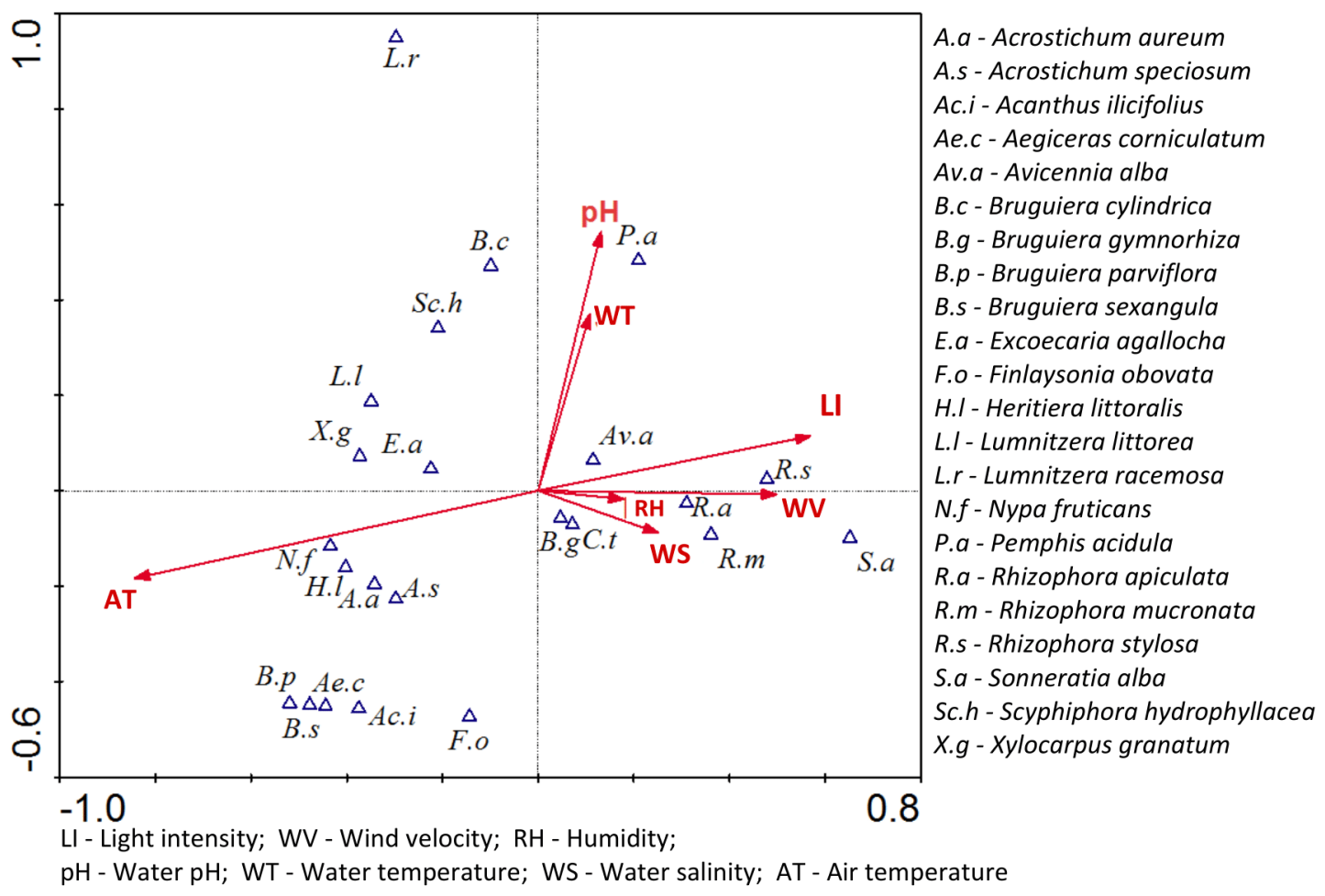

Figure 5. The interaction of environmental factors with the species of mangrove flora analyzed using Canonical Correspondence Analysis (CCA). The graph of CCA shows the ordination of species and environmental factors. Triangle symbols: mangrove species, arrow lines: environmental factors

Analysis of the interaction of environmental factors with the species of mangrove flora was done using Canonical Correspondence Analysis (CCA). The results of the Monte Carlo permutation test showed that all environmental factors measured in this study had significant $(\mathrm{p}<0.05)$ interaction with the mangrove flora community except for the water $\mathrm{pH}(\mathrm{p}>0.05)$, (Figure 5). Salinity, air humidity and wind velocity had influence on $R$. apiculata, $R$. mucronata, $R$. stylosa and $S$. alba. These species are the dominant species that grow closest to the sea and are able to survive in inundated substrate conditions for long time (Crase et al. 2013). Species $X$. granatum, E. agallocha, B. cylindrica, B. parviflora, Heritiera, Lumnitzera, Scyphiphora and Nypa were mostly found in zones adjacent to land zone. These species grow more tolerant to shorter seawater inundation. For other species, the effect of salinity is lower for the mangrove flora on the landward side.

\section{Zonation of mangrove in Belitung Island}

The zonation of mangrove flora is the ability of mangrove plant species to grow in specific substrates or zones that are most suitable for their growth (plasticity). Its zonation is also influenced by local topographic contours that form vegetation patterns (Tomlinson 1994)

The result showed that the mangrove forest in Kuale Tambak was located behind the beach casuarina vegetation (Figure 6). The Kuale Tambak coastal area was overgrown by terrestrial plant vegetation, while the mangrove forest was under the tidal influence of the watershed which boils down to the Manggar River. The zonation in the Kuale Tambak Beach from the coast to the mainland was coastal forest vegetation, such as Scaevola taccada, Pandanus tectorius and Casuarina equisetifolia. Then, closer to the mangrove forest, there was Acacia mangium, where $S$. taccada and Pandanus tectorius were found again. The mangrove vegetation began with $R$. apiculata, Lumnitzera racemosa, B. cylindrica and $R$. stylosa. Furthermore, $R$. mucronata, $R$. apiculata grow together with Sonneratia alba, B. gymnorhiza and Ceriops tagal. Near the riverbank, there were $R$. apiculata, $R$. stylosa, S. alba, B. gymnorhiza and Avicennia alba. Across the river was also covered by R. apiculata, $R$. mucronata and S. alba.

Asam Beach had mangrove vegetation in the intertidal zone. The seaward facing zones were covered by Sonneratia alba, R. mucronata, R. apiculata and Pemphis acidula (Figure 7). At the back of that species were $R$. stylosa, B. gymnorhiza, Ceriops tagal and R. apiculata. Furthermore, $R$. apiculata, $R$. Mucronata, Lumnitzera littorea, Bruguiera gymnorhiza and Ceriops tagal species still grow well. Approaching to the border with landward, there were Xylocarpus granatum, Heritiera littoralis, Scyphiphora hydrophyllacea, B. gymnorhiza and $R$. apiculata also Ceriops tagal species. The mangrove forest floor in the backside was covered by mangrove ferns (Acrostichum speciosum). At the border of mangrove forests and land vegetation were Podocarpus neriifolius, Hibiscus tiliaceus and Pandanus tectorius. 


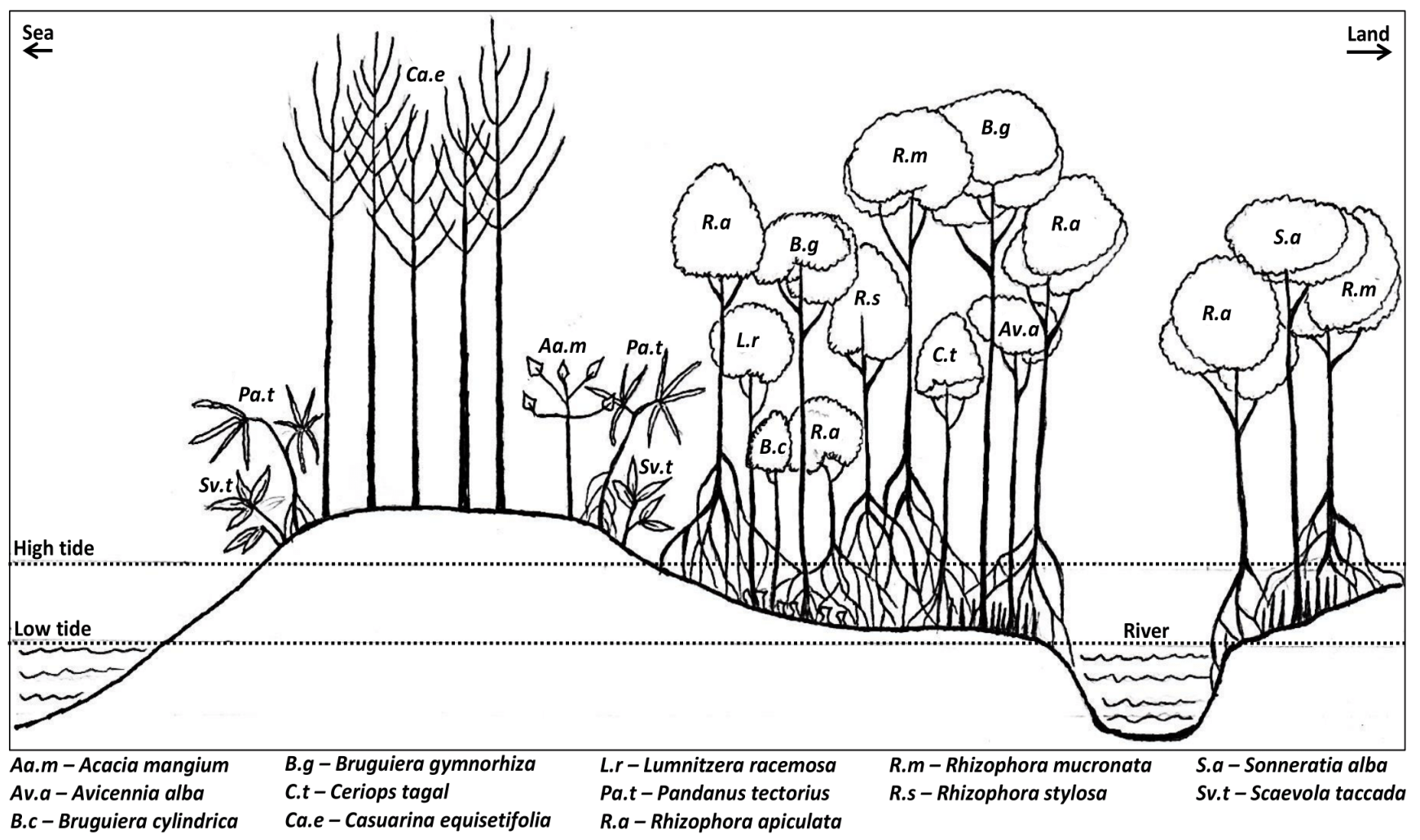

Figure 6. Schematic cross-section of coastal area in Kuale Tambak beach, Belitung Island, Indonesia

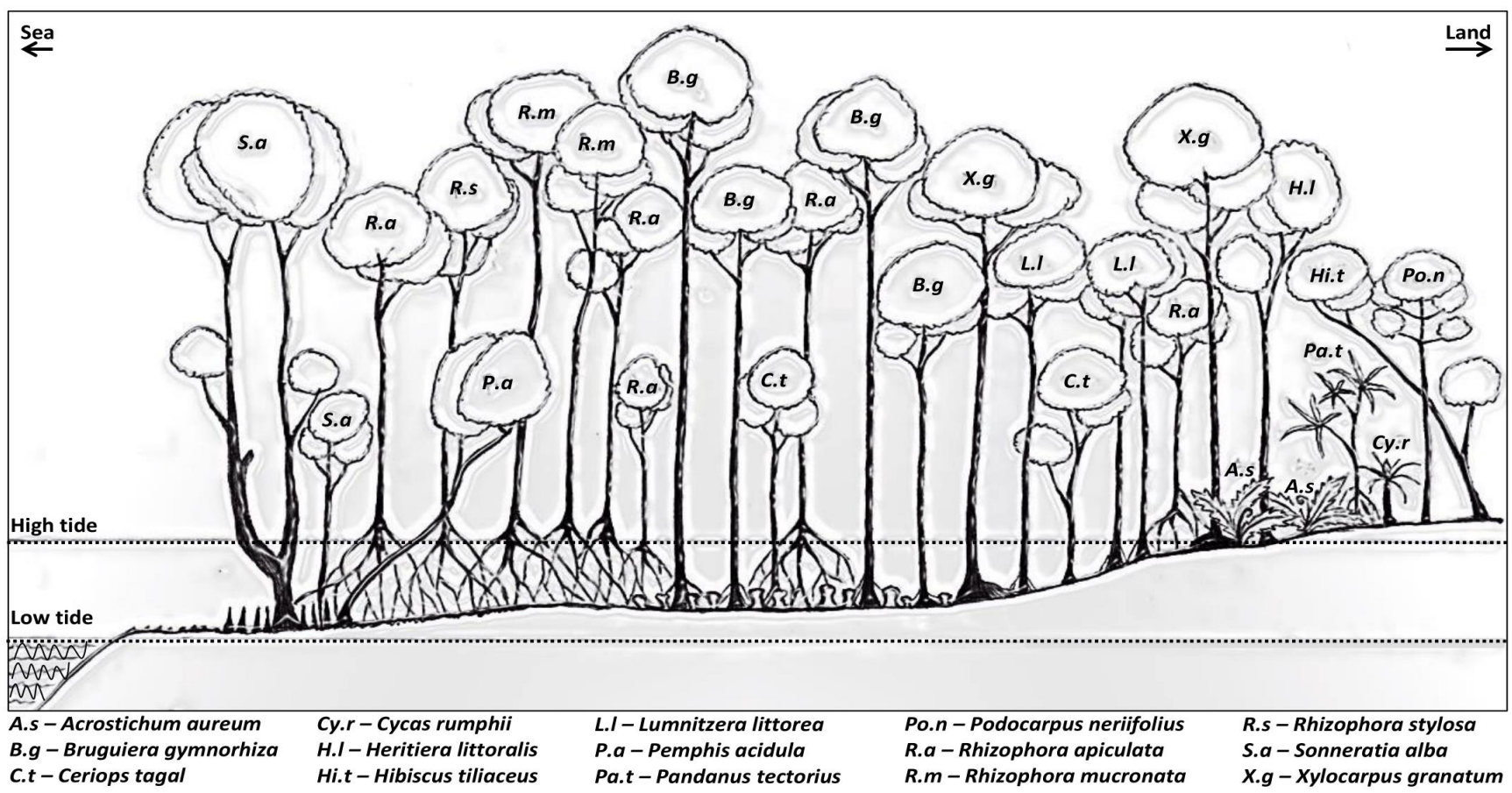

Figure 7. Schematic of mangrove flora zonation in Asam beach, Belitung Island, Indonesia 


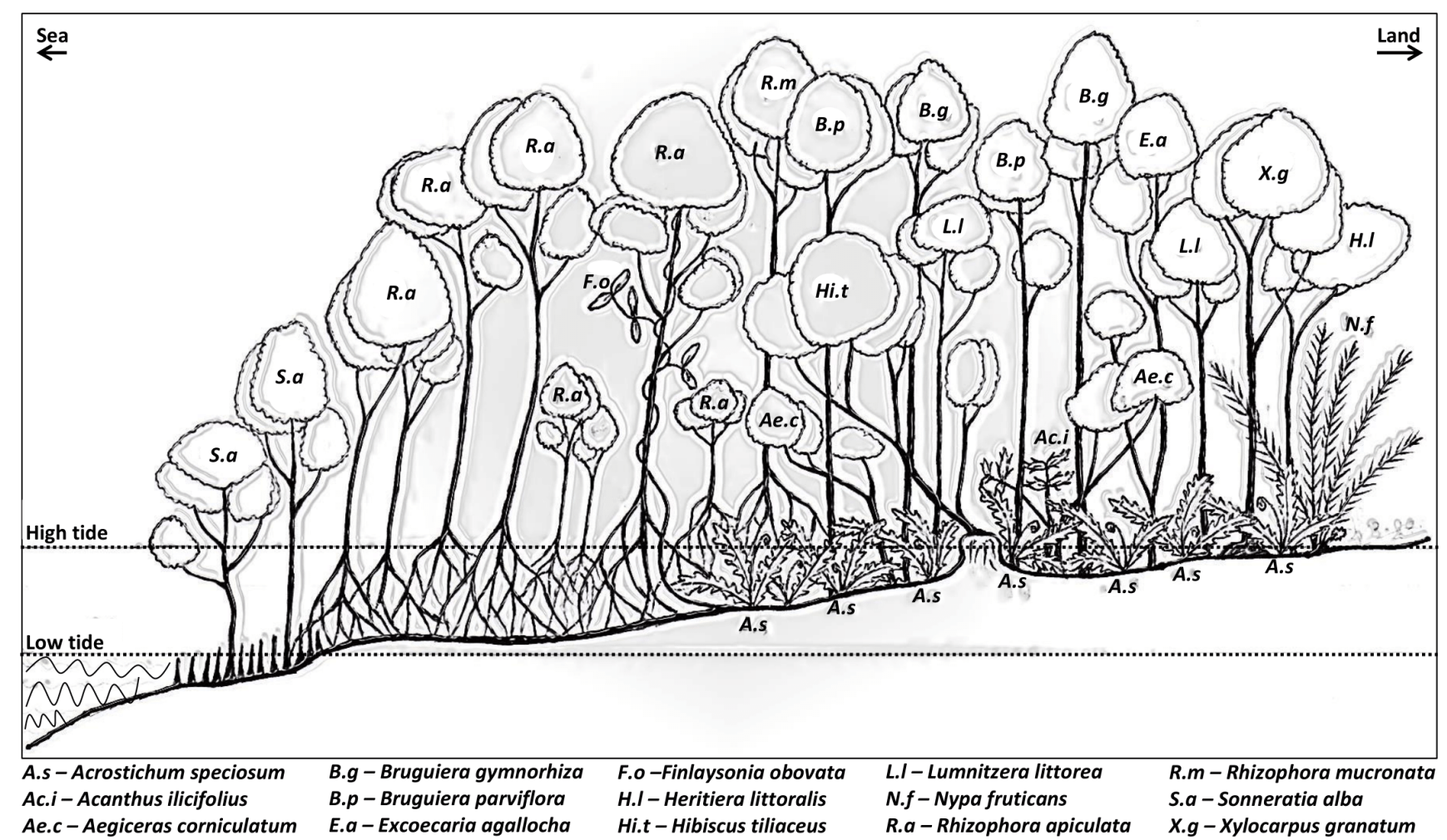

Figure 8. Schematic of mangrove flora zonation in Kembiri beach, Belitung Island, Indonesia

The mangrove forest in Kembiri Beach was in intertidal zone adjacent to the estuarine of the Kembiri River. The mangrove forest in seaward facing zones was covered by Sonneratia alba and dominated by $R$. apiculata (Figure 8). Furthermore, there was the Rhizophora zone (R. apiculata and $R$. mucronata). Then there were a climber mangrove species Finlaysonia obovata, that climbed on $R$. apiculata. In their backside was $B$. parviflora, Aegiceras corniculatum and $R$. apiculata. Furthermore, to the landward border, there were Lumnitzera littorea, $B$. gymnorhiza, Excoecaria agallocha, Nypa fruticans and Xylocarpus granatum. The forest floor was dominated by mangrove ferns (Acrostichum speciosum). Also, there was the associate mangrove species (Hibiscus tiliaceus) which grows on higher ground mounds in mangrove area.

Mangrove vegetation in Indonesia generally consists of 5 flora groups, namely Rhizophora, Sonneratia, Bruguiera, Avicennia and Nypa (Kusmana 2014). Rhizophora apiculata can grow in the front to the back of the mangrove forest. Rhizophora apiculata is tolerant species and able to dominate in mangrove forest areas (Giesen et al. 2007). The species of Rhizohopra stylosa was not found in the mangrove area of the Kembiri Beach, but grew well in the Kuale Tambak and Asam beaches. Rhizophora stylosa prefers sandy substrate (Kusmana 2014), while the substrate at Kembiri was dominated by mud, so these species were not found. It was very rare to find Avicennia species in the Kembiri and Asam sites. Avicennia was more common in the Belitung Mangrove Park area. The substrate in Belitung Mangrove Park was sandy with some mud on seaward facing side and always inundated which is very suitable for Avicennia. Avicennia species are usually found on the seaward side with submerged sandy substrates or in shallow sandy estuaries with low salinity water (Giesen et al. 2007). The Asam Beach substrate, which was dominated by coral rock and deep muddy in the Kembiri, it is not potential for Avicennia to grow properly. The difference in zonation patterns of mangrove flora is caused by local topography on Belitung Island, substrate conditions and salinity factors on the mangroves of Belitung Island.

In conclusion, the diversity of mangrove flora in Belitung Island consists of 24 species belonging to 16 genera and 12 families. Rhizophoraceae dominates the mangrove vegetation, consisted of Bruguiera (4 species), Rhizophora (3 species) and Ceriops (1 species). The zonation of mangrove flora in every location shows a different zonation pattern. This pattern is influenced by local topography, substrate conditions and salinity factors that affect the ability to grow in each species of mangrove flora. The species of mangrove flora that are tolerant of salinity are able to grow in areas with seawater inundation for long time, while the intolerant species more able to grow in landward areas.

\section{ACKNOWLEDGEMENTS}

We would like to express a high appreciation and thank Akbar Alfarisyi from the Bangka-Belitung Biodiversity Observer (BBO) foundation, Chief of Kembiri Village, 
Kek Syari's family, Bimo Purnomo's family, Chief of Aik Kelik Village and Yudi from Forum Daerah Aliran Sungai (Fordas) for their support on this research.

\section{REFERENCES}

Akhrianti I. 2016. Mangrove ecosystems in Sebongkok Island, District of Selat Nasik, Belitung Timur District. J Aquatropica Asia 3 (1): 13-21.

Ardiansyah WI, Pribadi R, Soenardjo N. 2012. Struktur dan komposisi vegetasi mangrove di kawasan pesisir Pulau Sebatik, Kabupaten Nunukan, Kalimantan Timur. J Mar Res 1 (2): 203-215. DOI: 10.14710/jmr.v1i2.2039.

Baderan DWK, Utina R, Lapolo N. 2018. Vegetation structure, species diversity, and mangrove zonation patterns in the Tanjung Panjang Nature Reserve Area, Gorontalo, Indonesia. Int J App Biol 2 (2): 112. DOI: $10.20956 / \mathrm{ijab.v2i2.5752.}$

Basyuni M, Baba S, Kinjo Y, Oku H. 2012. Salinity increases the triterpenoid content of a salt secretor and a non-salt secretor mangrove. Aquat Bot 97: 17-23. DOI: 10.1016/j.aquabot.2011.10.005.

Bunt JS, Williams WT. 1981. Vegetational Relationships in the mangroves of tropical Australia. Mar Ecol Progr Ser 4: 349-359. DOI: 10.3354/meps004349.

Chen L, Tam NFY, Wang W, Zhang Y, Lin G. 2013. Significant niche overlap between native and exotic Sonneratia mangrove species along a continuum of varying inundation periods. Estuar Coast Shelf Sci 117:22-28. DOI: 10.1016/j.ecss.2012.09.009.

Costa LD, Budiastuti MTHS, Sutrisno J, Sunarto. 2019. The diversity of plant species in a mangrove forest in the Coast of Metinaro, TimorLeste. Earth Environ Sci 256: 1-12. DOI: 10.1088/17551315/256/1/012026.

Crase B, Liedloff A, Vesk PA, Burgman MA, Wintle BA. 2013. Hydroperiod is the main driver of the spatial pattern of dominance in mangrove communities. Global Ecol Biogeogr 22 (7): 806-817. DOI: 10.1111/geb.12063.

Duke NC, Kudo H. 2018. Bruguiera $\times$ dungarra, a new hybrid between mangrove species $B$. exaristata and B. gymnorhiza (Rhizophoraceae) recently discovered in north-east Australia. Blumea 63(3): 279-285. DOI: 10.3767/blumea.2018.63.03.03.

FAO. 2007. The World's Mangroves 1980-2005. Food and Agriculture Organization, Rome, Italy. http://www.fao.org/3/a1427e/a1427e00.htm

Firmansyah, Satjapradja O, Supriono B. 2013. Potensi dan komposisi vegetasi pada ekosistem hutan mangrove di Selat Nasik Kabupaten Belitung, Provinsi Kepulauan Bangka Belitung. Journal Nusa Sylva. 13 (2): 9-18. [Indonesian]

Giesen W, Wulffraat S, Zieren M, Scholten L. 2007. Mangrove Guidebook for Southeast Asia. Dharmasarn Co., Ltd. Bangkok, TH.

Hendy IW, Cragg M. 2017. Rhizophora stylosa prop roots even when damaged prevent wood-boring teredinids from toppling the trees. Hydrobiologia 803:333-344. DOI: 10.1007/s10750-017-3106-6.

Heriyanto NM, Subiandono E. 2012. Komposisi dan struktur tegakan, biomasa dan potensi kandungan karbon hutan mangrove di Taman Nasional Alas Purwo. J Penelitian Hutan dan Konservasi Alam (JPHKA) 9(1): 23-32. DOI: 10.20886/jphka.2012.9.1.023-032.

Hoppe-Speer SCL, Adams JB, Rajkaran A, Bailey D. 2011. The response of the red mangrove Rhizophora mucronata Lam. to salinity and inundation in South Africa. Aquat Bot 95: 71-76. DOI: 10.1016/j.aquabot.2011.03.006.

Islam AKMN, Haque AE, Maniruzzaman, Jamali T, Haque T, Alfasane MA, Nahar N, Jahan N, Sultana S, Kumar TS. 2019. Species distribution in different ecological zones and conservation strategy of halophyte of Sundarbans mangrove forest of Bangladesh. Sabkha Ecosyst Task Veget Sci 6 (30): 479-495. DOI: 10.1007/978-3-03004417-6 30.

Karyamsetty JH. 2017. Floral diversity, phenology, and pollination mechanism of true viviparous and crypto-viviparous mangroves of Godavari and Krishna Delta of Andhra Pradesh, India. Intl J Conserv Sci 8 (4): 723-730.

Krauss KW, McKee KL, Lovelock CE, Cahoon DR, Saintilan N, Reef R, Chen L. 2014. How mangrove forests adjust to rising sea level. New Phytol 202: 19-34. DOI: 10.1111/nph.12605.

Kusmana C. 2014. Distribution and current status of mangrove forests in Indonesia. In: Faridah-Hanum I, Latiff A, Hakeem KR, Ozturk M (eds.). Mangrove Ecosystems of Asia: Status, Challenges and Management Strategies. Springer Science Business Media. New York, USA.

Liu M, Zhang H, Lin G, Lin H, Tang D. 2018. Zonation and directional dynamics of mangrove forests derived from time-series satellite imagery in Mai Po, Hongkong. Sustainability 10 (1913): 1-16. DOI: 10.3390/su10061913

Magurran AE. 1988. Ecological Diversity and Its Measurement. Princeton University Press. New Jersey, USA.

Martinez MR, Buot Jr IE. 2018. Mangrove assessment in Manamoc Island for coastal retreat mitigation. J Mar Island Cult 7 (1): 65-83. DOI: 10.21463/jmic.2018.07.1.05.

Mughofar A, Masrkuri M, Setyono P. 2018. Zonasi dan komposisi vegetasi hutan mangrove Pantai Cengkrong, Desa Karanggandu, Kabupaten Trenggalek, Jawa Timur. J Pengelolaan Sumberdaya Alam dan Lingkungan 8 (1): 77-85. DOI: 10.29244/jps1.8.1.77-85.

Noor YR, Khazali M, Suryadiputra INN. 2012. Panduan Pengenalan Mangrove di Indonesia. Wetland International-Indonesia Programme, Bogor. [Indonesian]

Phillips EA. 1959. Methods of Vegetation Study. Holt, Rinehart and Winston, Inc. New York, USA.

Ridho MS, Yustiati A, Riyantini I. 2015. Keanekaragaman dan sebaran mangrove di kawasan pulau-pulau kecil Kecamatan Manggar, Kabupaten Belitung Timur. Jurnal Perikanan dan Kelautan 3: 1-10.

Rugayah, Retnowati A, Windadri FI, Hidayat A. 2004. Pengumpulan Data Taksonomi. In: Rugayah, Widjaja EA, Praptiwi (eds). Pedoman Pengumpulan Data Keanekaragaman Flora. Pusat Penelitian Biologi, Lembaga Ilmu Pengetahuan Indonesia, Bogor. [Indonesian]

Tomlinson PB. 1994. The Botany of Mangroves. Cambridge University Press. Cambridge, UK.

Ulumuddin YI, Setyawan AD. 2017. Mangrove forest exploration of Tambelan and Serasan Islands: Species composition, mapping of mangrove forest distribution and threat potential. Pros Sem Nas Masy Biodiv Indon 3: 45-55. DOI: 10.13057/psnmbi/m030109. [Indonesian]

Umroh, Adib W, Sari SP. 2016. Detection of mangrove distribution in Pongok Island. Procedia Environ Sci 33:253-257. DOI: 10.1016/j.proenv.2016.03.076.

Wilhm JL, Dorris TC. 1968. Biological parameters for water quality criteria. BioScience 18 (6): 477-481.

Yuningsih E, Simbala HEI, Kandou FEP, Saroyo. 2013. Keanekaragaman vegetasi mangrove di Pantai Tanamon, Sulawesi Utara. J Bios Logos 2(3): 78-84. DOI: $10.35799 /$ jbl.3.2.2013.4434. 